\title{
Reduced spontaneous apoptosis in peripheral blood neutrophils during exacerbation of COPD
}

\author{
M.W.R. Pletz ${ }^{*, \#,+}$, M. loanas*,+, A. de Roux*, O. Burkhardt*, ${ }^{*}$, H. Lode*
}

Reduced spontaneous apoptosis in peripheral blood neutrophils during exacerbation of COPD. M.W.R. Pletz, M. Ioanas, A. de Roux, O. Burkhardt, H. Lode. (C)RS Journals Ltd 2004.

ABSTRACT: A major feature of acute exacerbation of chronic obstructive pulmonary disease (COPD) is the accumulation of activated neutrophils in the bronchial tree. This phenomenon can be explained by an increased migration and/or by a prolonged survival due to an inhibition of spontaneous apoptosis. The aim of this study was to assess the apoptotic behaviour of peripheral blood neutrophils in COPD patients during an acute exacerbation.

Thirty-six hospitalised COPD patients with an acute exacerbation and 10 healthy volunteers were included. Blood samples were obtained at admission, after 3-5 days and at discharge. Spontaneous apoptosis of isolated neutrophils was measured based on Annexin V-PE binding and nuclear morphology after culturing for $18 \mathrm{~h}$.

At admission, significantly lower rates of spontaneous apoptosis were noted in COPD patients compared with healthy volunteers (mean \pm SD $31 \pm 13 \%$ versus $44 \pm 18 \%$ ). The mean percentages of apoptotic neutrophils were $31 \pm 13 \%$ at admission, $39 \pm 15 \%$ after 3-5 days and $47 \pm 18 \%$ at discharge. There was a statistically significant difference between the rates of spontaneous apoptosis on the first day and at discharge. Neither forced expiratory volume in one second $<35 \%$ predicted, smoking habit, corticosteroid therapy nor evidence of bacterial infection showed any influence on the spontaneous apopotosis in this study.

In conclusion, during acute exacerbations of chronic obstructive pulmonary disease, neutrophil granulocytes show a reduced spontaneous apoptosis that increases progressively after treatment and clinical remission. This raises the question of the importance of neutrophil apoptosis in the development and resolution of exacerbations of chronic obstructive pulmonary disease.

Eur Respir J 2004; 23: 532-537.
*Dept of Chest and Infectious Diseases, City Hospital Emil von Behring affil. Freie Universitaet Berlin, Germany. ${ }^{\#}$ Dept of International Health, Rollins School of Public Health, Emory University, Atlanta, USA. "Dept of Chest and Intensive Care Medicine, Otto-vonGuericke Universitaet, Magdeburg, Germany.

Correspondence: H. Lode, Dept of Chest and Infectious Diseases, Zentralklinik E. v. B/Dept. Lungenklinik Heckeshorn, Zum Heckeshorn 33, 14109 Berlin, Germany.

Fax: 493080022623

E-mail: haloheck@zedat.fu-berlin.de

Keywords: Apoptosis

chronic obstructive pulmonary disease

exacerbation

flow cytometry

neutrophils

Received: July 312003

Accepted after revision: November 242003

${ }^{+}$Authors contributed equally to this paper. M.W.R. Pletz was supported by the German Research Foundation. M. Ioanas is a fellow of the Paul-Ehrlich-Gesellschaft 2002
The pathogenesis of acute exacerbations of chronic obstructive pulmonary disease (COPD) is not fully understood. Viral and bacterial infection and airway inflammation are the main contributors to a cycle of events that result in tissue damage and progressive bronchial obstruction [1].

Massive accumulation of activated neutrophils in the bronchial tree is a frequent finding in the bronchoalveolar lavage (BAL) of COPD patients in both stable state and exacerbation [2]. This phenomenon could be explained by two mechanisms: 1) an increased neutrophil migration; and/or 2) a prolonged survival due to inhibition of spontaneous apoptosis [3]. During the resolution of inflammation, neutrophils are removed from inflammatory sites by spontaneous apoptosis, leading to their recognition and phagocytosis by macrophages [4]. Any significant inhibition of neutrophil apoptosis can lead to excessive neutrophil accumulation and damage to healthy tissue [5]. This concept is supported by several studies showing reduced apoptosis of blood and/or BAL neutrophils in both acute respiratory distress syndrome (ARDS) [6] and pneumonia [7].

Deregulation of apoptosis of various types of cells appears to have an important role in the pathogenesis and progression of COPD. Increased serum levels of soluble Fas receptor, which is an inhibitor of apoptosis [8], have been associated with severe COPD [9]. However, little is known about the apoptotic features of neutrophils during acute exacerbations of COPD.

Since neutrophils are the main inflammatory cells in an acute exacerbation of COPD, reduced spontaneous apoptosis of the peripheral blood neutrophils during an exacerbation is expected.

\section{Patients and methods}

\section{Study subjects}

Thirty-six hospitalised patients with documented diagnosis of COPD according to GOLD-criteria [2] and presenting with an acute exacerbation were included. The acute exacerbation was defined by any combination of the following criteria: worsening dyspnoea, increase in sputum volume and increase in sputum purulence. Patients with a new radiological infiltrate, known HIV infection, evidence of bronchiectasis or tumour, and/or immunosuppressive therapy were excluded. Treatment of acute exacerbation consisted of inhaled bronchodilators, systemic corticosteroids (prednisolone $75 \mathrm{mg}$ i.v. daily for 3 days, followed by oral therapy for 2 weeks), oxygen in case of hypoxaemia and antibiotics in case 
of purulent sputum and/or evidence of microbial growth in sputum $(\beta$-lactam/ $\beta$-lactamase inhibitor combination or fluoroquinolones).

In order to ensure a reference figure for the apoptotic process, a nonmatching group of 10 healthy, nonsmoking volunteers from the medical staff were also investigated. Except oral contraceptives in individual cases, no medication was taken by subjects of this group. The study was approved by the Ethics committee of the Freie Universität Berlin (Berlin, Germany) and written informed consent was obtained from all patients and volunteers.

\section{Study design}

To investigate the apoptotic behaviour of peripheral blood neutrophils in COPD patients during an acute exacerbation, a prospective study was conducted over a period of 18 months at the Chest Hospital Heckeshorn, Berlin (Germany).

All patients were investigated at admission, after 3-5 days and at discharge (usually after 14 days). Demographic and clinical data, smoking habits, lung function tests, and microbiological findings were collected from the patient chart. In case of hospital stay shorter than 14 days, an ambulatory visit was scheduled. At each visit, venous heparin blood samples $(20 \mathrm{~mL})$ were obtained and immediately processed in the laboratory. The proportions of apoptotic neutrophils after culturing for $18 \mathrm{~h}$ were compared between those three visits.

Additionally, the authors investigated if there is a correlation between clinical parameters and the apoptotic behaviour of the neutrophils on admission. Therefore, COPD patients were grouped according to forced expiratory volume in one second (FEV1), confirmation of bacterial infection, body mass index (BMI), and the spontaneous apoptosis rates were compared between those groups.

From the healthy volunteers, three consecutive blood samples were collected on different days. The mean value of the three measurements has been used in the analysis.

\section{Methods}

Neutrophils are known to undergo readily spontaneous apoptosis both in vivo and in vitro. For each blood sample, apoptotic neutrophils were measured twice: immediately after obtaining the blood sample (baseline apoptosis) and after culturing for $18 \mathrm{~h}$. The spontaneous apoptosis rate represents the proportion of spontaneous apoptotic neutrophils measured after $18 \mathrm{~h}$ culturing minus baseline value and was considered for further analysis. Two different methods were used: 1) flow cytometric measurement of Annexin V-PE/7AAD-stained cells, and 2) light microscopy to assess the nuclear morphology.

Separation of neutrophils and culture. After adding $10 \mathrm{~mL} 6 \%$ dextran and a 1 -h sedimentation period $\left(37^{\circ} \mathrm{C}\right)$, neutrophils were isolated from the blood samples by density gradient centrifugation using Ficoll (Biochrom KG, Berlin, Germany), followed by a hypotonic lysis of erythrocytes. Cells were washed and resuspended at a concentration of $1 \times 10^{6}$ cells $\cdot \mathrm{mL}^{-1}$ in RMPI medium $(\mathrm{pH}=7.4)$, supplemented with $2.8 \mathrm{~g} \cdot \mathrm{L}^{-1}$ HEPES, $1 \mathrm{~g} \cdot \mathrm{L}^{-1} \mathrm{NaHCO}_{3}$ and $10 \%$ foetal calf serum. Spontaneous apoptosis was measured after culturing the cells for $18 \mathrm{~h}$ at $37^{\circ} \mathrm{C}, 5 \%$ carbon dioxide $\left(\mathrm{CO}_{2}\right)$ and $90 \%$ relative humidity.

Flow cytometry. Isolated neutrophils were stained with Annexin V-PE/7-AAD with the Annexin V-PE Apoptosis
Detection Kit I (PharMingen; Becton Dickinson, Heidelberg, Germany), according to manufacturer's protocol. Briefly, cells were washed and centrifuged in binding buffer (0.1 M HEPES/ $\mathrm{NaOH}$ (pH 7.4), 1.4 M NaCl, $25 \mathrm{mM} \mathrm{CaCl}$ ), and the pellet was resuspended in $5 \mu \mathrm{L}$ Annexin V-PE, $5 \mu \mathrm{L} 7$ AAD (7amino-actinomycin D) and $100 \mu \mathrm{L}$ binding buffer, followed by incubation for $15 \mathrm{~min}$ at room temperature in the dark. Measurement was performed by a three-colour flow-cytometer (FACScan; Becton-Dickinson) using CellQuest software. A predetermined count of 10,000 granulocytes was set on forward light scatter. Annexin V-PE was measured in FL-2 and 7-AAD in FL-3. Only cells with an intact cell membrane, i.e. 7-AAD (-) and Annexin V-PE (+), were considered as apoptotic.

Light microscopy. A cytospin slide was prepared from the RMPI-suspended neutrophils and stained with Pappenheim (Sigma, Taufkirchen, Germany) solution. Two-hundred cells were counted and morphologically evaluated by light microscopy. Apoptotic neutrophils were identified as having singlelobed or completely fragmented nuclei with intense nuclear staining, suggesting chromatin condensation [6].

\section{Statistics}

The sample size was subjectively set between 30 and 40 , since no available reference data could allow a statistical estimation. Spontaneous apoptosis rates were expressed in percentage of Annexin V-PE (+), 7-AAD (-) cells (measured by flow cytometry) and percentage of apoptotic neutrophils (apoptotic neutrophils $\times 100 /$ total neutrophils) when counted by light microscopy (nuclear morphology). The parametric distribution of the data was confirmed by the Shapiro-Wilk test for small samples $(n \leqslant 50)$. Correlation between the two methods of measurement was evaluated by Pearson's coefficient. Spontaneous apoptosis rates at each visit were contrasted using paired t-test tests. Multiple group comparisons were performed using analysis of variance for repeated measures.

Additionally, COPD patients were grouped according to clinical parameters, and the spontaneous apoptosis rates on admission between those groups, as well as between COPD patients and healthy volunteers, were compared using unpaired t-tests. Results are expressed as percentage and mean \pm SD. A p-value of $<0.05$ was considered as significant.

\section{Results}

\section{Demographic data}

Thirty-six COPD patients with acute exacerbation were enrolled in the study. The demographic characteristics are shown in table 1 . Additionally, 10 nonmatching, healthy, nonsmoking volunteers from the medical staff were analysed (four male, six female).

\section{Correlation between the methods used for measuring apoptosis}

The two independent methods used for the measurement of apoptosis (nuclear morphology and Annexin V-PE/7-AAD staining) showed a good correlation ( $r=0.711$, fig. 1$)$ on a significant level $(\mathrm{p}<0.0001)$. Overall, the values provided by flow cytometry were discretely higher compared to the data obtained by judging the nuclear morphology by light 
Table 1.-Baseline characteristics of chronic obstructive pulmonary disease patients

\begin{tabular}{lc}
\hline Subjects n & 36 \\
Age yrs & $69.3 \pm 7.3$ \\
M/F n & $22 / 14$ \\
Smoking habits $\mathrm{n}^{\#}$ & $8 / 22 / 6$ \\
Pack-years & $39.8 \pm 18.3$ \\
BMI & $25.3 \pm 6.1$ \\
Corticosteroid therapy & \\
$\quad$ Oral & $13(36.1)$ \\
$\quad$ Inhaled & $12(33.3)$ \\
FEV1\% pred on admission & $41.7 \pm 17.1$ \\
Microbial growth in sputum & $13(36.1)$ \\
Severity of acute exacerbation & \\
$\quad$ Mild & $9(25.0)$ \\
Moderate & $10(27.7)$ \\
Severe & $17(47.2)$ \\
\hline
\end{tabular}

Data are presented as mean \pm SD or $n(\%)$ unless otherwise stated. $\mathrm{M}$ male; F: female; BMI: body mass index (weight $(\mathrm{kg}) /$ height ${ }^{2}(\mathrm{~cm})$ ); FEV1: forced expiratory volume in one second. ${ }^{\#}$ : smokers/ex-smokers/ nonsmokers.

microscopy. As an example, figure 2 presents a cytospin preparation of isolated neutrophils and figure 3 shows flow cytometry density blots of neutrophils stained with Annexin V-PE/7-AAD before and after culturing for $18 \mathrm{~h}$.

Spontaneous apoptosis rates in COPD patients during an acute exacerbation and in healthy volunteers

The measurement of spontaneous neutrophil apoptosis in hospitalised COPD patients with acute exacerbation on admission, after 3-5 days and at discharge (day 14 \pm 2 ) showed increasing values (fig. 4) for both independent methods, flow cytometry and light microscopy. Induced apoptosis rates of COPD patients and healthy volunteers are presented in table 2 . Statistical analysis revealed significant differences of spontaneous apoptosis rates in COPD patients between admission and day 3-5 ( $\mathrm{p}=0.006)$, as well as between admission and discharge $(\mathrm{p}<0.0001)$. The differences between the three time-points were also confirmed by multigroup comparison using analysis of variance for repeated measures $(\lambda=0.357, \quad \mathrm{p}<0.0001)$. Compared with COPD patients, the spontaneous apoptosis rates in the nonmatched healthy volunteers were significantly higher than the values

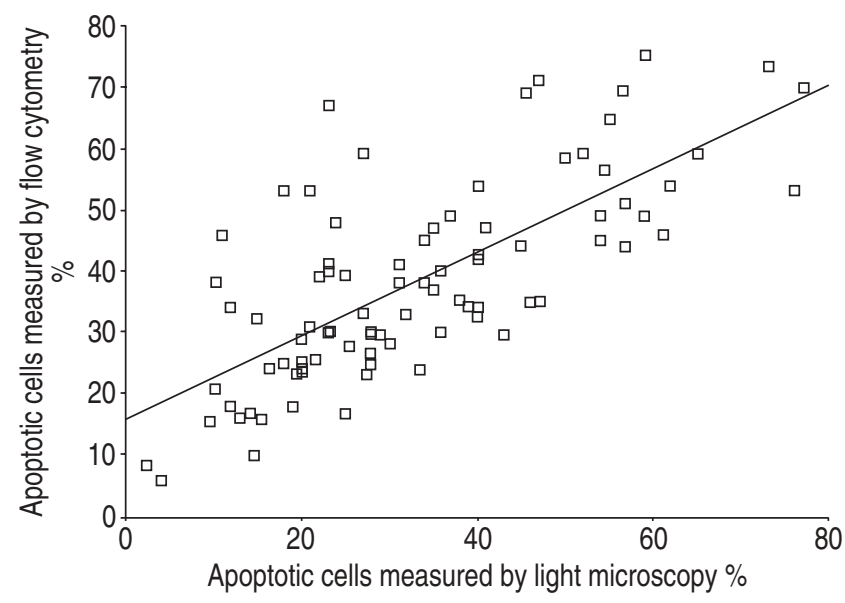

Fig. 1.-Correlation between the two independent methods (flow cytometry: binding of Annexin V-PE; light microscopy: nuclear morphology) for detection of apoptotic neutrophils. $\mathrm{r}=0.711$, $\mathrm{p}<0.0001$.
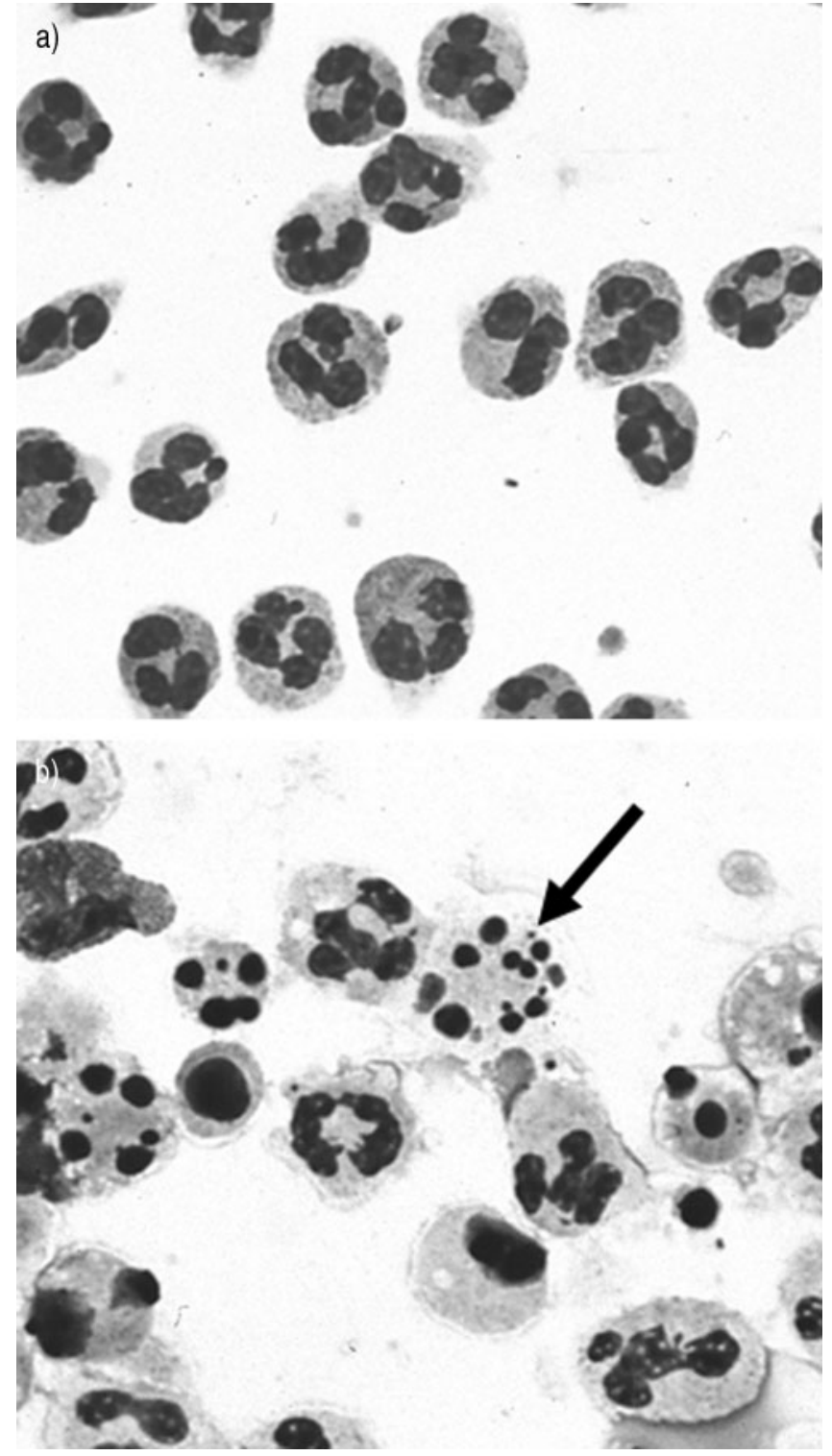

Fig. 2. - Light microscopy. a) Normal neutrophils immediately after obtaining the blood sample. Usually no apoptotic cells are seen. b) Neutrophils after $18 \mathrm{~h}$ of culture. Arrow: an apoptotic cell with condensed nuclear fragments.

obtained at admission (43.9 versus $31.2 \%, \mathrm{p}=0.01$ ), but there was no significant difference when compared to values obtained at discharge (fig. 4).

Correlation of spontaneous neutrophil apoptosis with clinical parameters in patients with COPD

COPD patients were grouped according to clinical parameters, and the induced apoptosis rate on admission was compared between those groups. No difference was found between patients for FEV $1<35 \%$ predicted at admission (FEV1 $<35 \% 31.5 \pm 13.7$ versus $\mathrm{FEV} 1>35 \% 32.6 \pm 11.8$, $\mathrm{p}=0.8$ ), $\mathrm{BMI}<20(\mathrm{BMI}<2034.2 \pm 13.0$ versus $\mathrm{BMI}>20$ 31.2 \pm 12.0 , $\mathrm{p}=0.6$ ), positive sputum culture (positive $34 \pm 12.1$ versus negative 25.6 $\pm 9.3, \mathrm{p}=0.06$ ), smoking habit (current smokers $33.9 \pm 13.5$ versus ex- and nonsmokers $30.8 \pm 12.3, p=0.5)$, and corticosteroid therapy on admission (inhaled $30.9 \pm 10.7$ versus oral $30.7 \pm 13.5, \mathrm{p}=0.9$ ). 

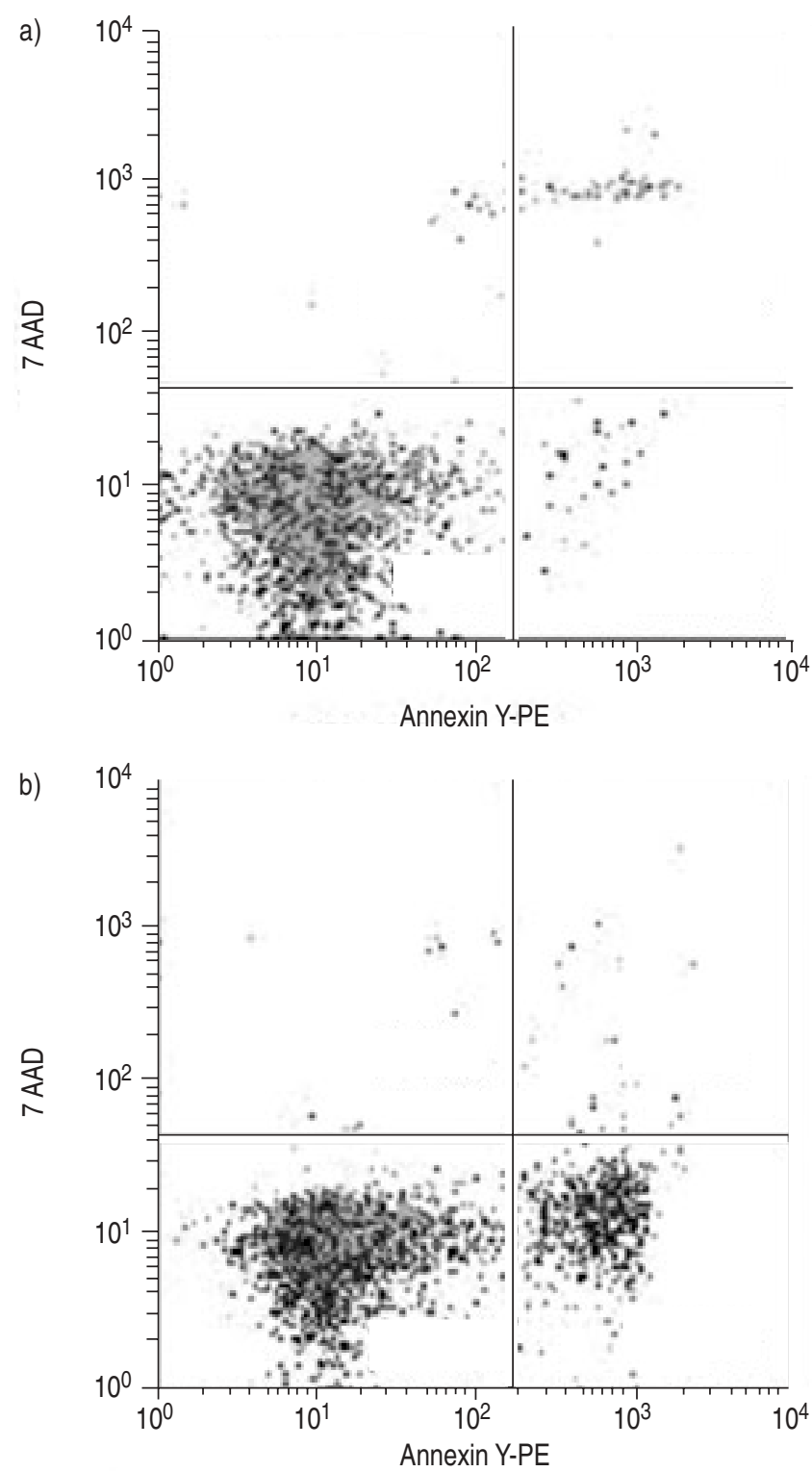

Fig. 3.-Flow cytometry. Isolated neutrophils before (a) and after culturing for $18 \mathrm{~h}$ (b). The right lower quadrant (RLQ) contains early apoptotic neutrophils with positive Annexin V-PE binding and negative 7-AAD binding (Annexin V-PE (+), 7-AAD (-)). Vital neutrophils in the lower left quadrant (LLQ) are Annexin V-PE (-), 7-AAD (-). Upper right quadrant (RUQ) contains late apoptotic and necrotic neutrophils (Annexin V-PE $(+)$, 7-AAD $(+)$ ). The amount of cells in each quadrant, expressed as $\%$ total cell count $(10,000)$, is as follows: LUQ: $0.4 \%$, b) $0.4 \%$; RUQ: a) $1.4 \%$, b) $0.7 \%$; LLQ: a) $97 \%$, b) $82.3 \%$; RLQ: a) $1.1 \%$, b) $16.55 \%$.

Serum levels of interleukin (IL)-6 and IL-8 were also measured by ELISA technique in a small group $(n=8)$ of COPD patients (at admission, after 3-5 days and at discharge) $(n=4)$ and healthy volunteers $(n=4)$. No statistical difference in IL-6 and IL-8 levels was observed during the course of acute exacerbation. Similarly there was no difference between COPD patients and healthy volunteers (data not shown).

\section{Discussion}

The present study identified a reduced spontaneous apoptosis of peripheral blood neutrophils in hospitalised

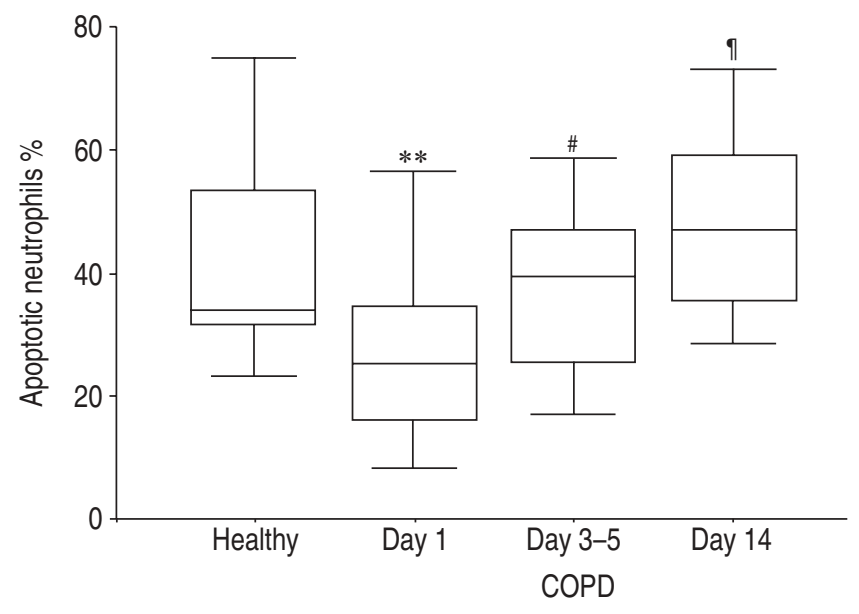

Fig. 4.-Spontaneous apopotosis rates of neutrophils in healthy volunteers and chronic obstructive pulmonary disease (COPD) patients during acute exacerbation (values provided by flow cytometry). ${ }^{* *}: \mathrm{p}=0.01$ (COPD day 1 versus healthy volunteers); ${ }^{\#}: \mathrm{p}=0.006$ (COPD day 3-5 versus COPD day 1); ${ }^{\circ}$ p $<0.0001$ (COPD day 14 versus COPD day 1$)$.

patients with an acute exacerbation of COPD. The spontaneous apoptosis rate increased progressively during the recovery.

In studies of this nature in which apoptosis is being measured, it is necessary to employ at least two independent methodologies to assay for programmed cell death [10]. The main features of apoptosis are the redistribution of phosphatidylserine from the inner to the outer layer of the cell membrane, and the condensation and fragmentation of chromatin [11]. The former can be assessed by binding of labelled Annexin V and the later by nuclear staining. Consequently, the current evaluation of apoptosis was based on flow-cytometric measurement of Annexin V-PE/7AAD-labelled neutrophils and morphological changes of the nucleus detected by light microscopy. In contrast to changes in nuclear morphology due to chromatin condensation and fragmentation, the externalisation of phophatidylserine is an early feature of apoptosis. Therefore, the apoptosis rates measured by Annexin V-PE/7-AAD staining were higher than those obtained by evaluating the nuclear morphology. However, there was a significant correlation between the two methods in this study and similar results were obtained with both methodologies.

The predominant cells accumulating in the lungs of COPD patients are neutrophils and it has been suggested that their prolonged survival could be accounted for by resistance to apoptosis [3, 12]. However, there is not much data available concerning the peripheral blood neutrophils compartment. The current authors hypothesised that peripheral blood neutrophils in acute exacerbation of COPD should also present a specific apoptotic pattern. This presumption is supported by the following evidence: 1) COPD is an inflammatory pulmonary disease with systemic impact [13]; and 2) cytokines and other signalling factors that suppress neutrophil apoptosis, such as soluble Fas [8] and IL-6 [14], have detectable levels in serum and plasma of COPD patients [9, 15]. YASUDA et al. [9] demonstrated that increased concentrations of soluble Fas, IL- 6 and tumour necrosis factor- $\alpha$ in plasma are independent risk factors for progression of COPD and correlate with the severity of the disease. However, soluble Fas-mediated suppression of apoptosis in neutrophils in particular has not yet been confirmed.

In a recent study, TURLEJ et al. [16] found an increased resistance to apoptosis in alveolar neutrophils from heaves-affected 
Table 2. - Total white blood cells (WBC), neutrophils (\%) and spontaneous apoptosis rates of neutrophils (\%)

\begin{tabular}{|c|c|c|c|c|c|c|}
\hline \multirow[t]{3}{*}{ Time point } & \multirow[t]{3}{*}{$\mathrm{WBC} \times 10^{6} \cdot \mu \mathrm{L}^{-1}$} & \multirow[t]{3}{*}{ Neutrophils } & \multicolumn{4}{|c|}{ Apoptotic neutrophils } \\
\hline & & & \multicolumn{2}{|c|}{ Flow cytometry } & \multicolumn{2}{|c|}{ Light microscopy } \\
\hline & & & Before incubation & After incubation & Before incubation & After incubation \\
\hline Admission & $10.2 \pm 2.8$ & $75.5 \pm 13.9$ & $1.3 \pm 0.6$ & $31.2 \pm 13.0$ & $0.2 \pm 0.6$ & $27.3 \pm 15.1$ \\
\hline After 3-5 days & $10.2 \pm 4.6$ & $75.7 \pm 10.0$ & $1.7 \pm 0.9$ & $39.0 \pm 14.6$ & $0.5 \pm 1.0$ & $32.6 \pm 17.4$ \\
\hline Day 14 & $10.6 \pm 2.7$ & $68.0 \pm 10.2$ & $2.1 \pm 0.9$ & $46.6 \pm 17.5$ & $0.4 \pm 1.0$ & $42.2 \pm 14.2$ \\
\hline
\end{tabular}

Data are presented as mean \pm SD.

horses; heaves is regarded to be an animal model for COPD or asthma [10]. The authors could not detect a significant difference in resistance to apoptosis of peripheral blood neutrophils. This was probably due to the small sample size (six healthy versus six heaves-affected horses). However, reduced spontaneous apoptosis of both local and peripheral blood neutrophils has already been reported in ARDS [6], severe inflammatory response syndrome [17] and pneumonia [7].

In this study there was a significantly reduced spontaneous apoptosis of neutrophils in COPD patients at the onset of the acute exacerbation compared to the time of discharge. Several factors can influence the apoptotic behaviour of neutrophils in COPD patients. Cigarette smoking is the most important risk factor for both development of COPD and COPDrelated mortality [2]. It has been demonstrated in vitro that nicotine prolongs neutrophil survival in a dose-dependent manner by suppressing apoptosis [18]. However, no clear correlation between smoking habits and neutrophil apoptosis could be found in the population studied here. This may be explained by the fact that nicotine has a short plasma half-life time and that the patients were either non/ex-smokers or current smokers who discontinued smoking in relation to the exacerbation, thus no immediate effect of nicotine on neutrophils could be observed.

Corticosteroids may also affect apoptosis. Corticosteroid therapy exhibits beneficial anti-inflammatory effects in some COPD patients in stable state or during acute exacerbation [2]. Although in vitro data suggest that high dosage of corticoids inhibit neutrophil apoptosis [19], it is likely that, in vivo, this mechanism is overwhelmed by inhibiting the antiapoptotic effect of various inflammatory cytokines. This could explain the paradox of constantly increasing apoptosis rates in the patients in this study despite the administration of intravenous and oral corticosteroids during hospital stay. An exclusion of patients on pre-hospital steroid therapy was not possible, since most of the patients with moderate or severe COPD depend on steroids.

In the population in this study, $36 \%$ of all patients (including those with no expectoration or without a valid sputum sample) presented with a microbial growth in sputum (nine Gram-negative and six Gram-positive pathogens). A microbial infection may be theoretically associated with decreased neutrophil apoptosis. In vitro studies show that administration of lipopolysacharide in rats results in massive activation and decreased apoptosis of neutrophils [20]. Common respiratory pathogens, such as Staphylococcus aureus and Pseudomonas aeruginosa, were also found to promote neutrophil survival in cell culture from patients with cystic fibrosis [21]. However, no differences in apoptosis level were found in patients with microbial growth compared with those with no growth. This observation may be explained by the fact that the patients presented no bacteraemia, so there was no direct effect of microbe or of endotoxaemia, as was the case in the in vitro studies.
A limitation of this study is the lack of thorough investigation of the cytokines that can affect apoptosis, such as IL-6 or granulocyte-macrophage colony-stimulating factor. The serum levels of two anti-apoptotic cytokines were measured in a small group of subjects, although no detectable difference between patients and healthy volunteers was found. Therefore, these measurements were not continued.

It is worth noting that in vivo findings can vary considerably in comparison to in vitro observations. This difference may be explained by the fact that, in vivo, neutrophils are exposed to several anti- and pro-apoptotic factors simultaneously under dynamic conditions, while in vitro studies are inherently limited to a small number of static interventions [6].

The reduced spontaneous apoptosis of peripheral blood neutrophils at the onset of the acute exacerbation and the subsequent recovery during the course of the disease shows that apoptosis of neutrophils is related to the course of exacerbation and might, therefore, be one pathogenic feature in this complex disease which has not yet been addressed. A number of arguments may be put forward to account for these findings: inhibition of neutrophil apoptosis at the onset of exacerbations might support the host in combating related infections, the decreased resistance to apoptosis at the end of the exacerbation might be part of the resolution of inflammation or the observations in this study may simply reflect the systemic effect of locally released inflammatory parameters. The current findings were definitively confirmed by two independent methods but could not be correlated to exacerbation-stimulating factors, such as microbial growth or smoking habits. This raises questions for further research addressing the pathogenic importance and reason for the reduced spontaneous apoptosis in neutrophils during exacerbations of chronic obstructive pulmonary disease.

Acknowledgements. The authors would like to thank M. Rau and G. Schreiber for expert technical assistance, and P.J. Halvey and L. McGee for critical reading of the manuscript.

\section{References}

1. Cole P. Host-microbe relationships in chronic respiratory infection. Respiration 1989; 55: Suppl. 1, 5-8.

2. Pauwels RA, Buist AS, Calverley PM, Jenkins CR, Hurd SS. Global strategy for the diagnosis, management, and prevention of chronic obstructive pulmonary disease. NHLBI/ WHO Global Initiative for Chronic Obstructive Lung Disease (GOLD) Workshop summary. Am J Respir Crit Care Med 2001; 163: 1256-1276.

3. Dibbert B, Weber M, Nikolaizik WH, et al. Cytokinemediated Bax deficiency and consequent delayed neutrophil apoptosis: a general mechanism to accumulate effector cells in inflammation. Proc Natl Acad Sci USA 1999; 96: 1333013335 . 
4. Savill JS, Wyllie AH, Henson JE, Walport MJ, Henson PM, Haslett C. Macrophage phagocytosis of aging neutrophils in inflammation. Programmed cell death in the neutrophil leads to its recognition by macrophages. J Clin Invest 1989; 83: 865-875.

5. Hallett MB, Lloyds D. Neutrophil priming: the cellular signals that say "amber" but not "green". Immunol Today 1995; 16: 264-268.

6. Matute-Bello G, Liles WC, Radella F, 2nd, et al. Neutrophil apoptosis in the acute respiratory distress syndrome. Am J Respir Crit Care Med 1997; 156: 1969-1977.

7. Droemann D, Aries SP, Hansen F, et al. Decreased apoptosis and increased activation of alveolar neutrophils in bacterial pneumonia. Chest 2000; 117: 1679-1684.

8. Cheng J, Zhou T, Liu C, et al. Protection from Fas-mediated apoptosis by a soluble form of the Fas molecule. Science 1994; 263: 1759-1762.

9. Yasuda N, Gotoh K, Minatoguchi S, et al. An increase of soluble Fas, an inhibitor of apoptosis, associated with progression of COPD. Respir Med 1998; 92: 993-999.

10. Kelly MG, Elborn JS, Kelly MG, Brown V, Ennis M. Measuring granulocyte apoptosis in airway inflammation. Thorax 2002; 57: 376.

11. Hale AJ, Smith CA, Sutherland LC, et al. Apoptosis: molecular regulation of cell death [published erratum appears in Eur J Biochem 1996 1; 237: 884]. Eur J Biochem 1996; 236: 1-26.

12. Mecklenburgh K, Murray J, Brazil T, Ward C, Rossi AG, Chilvers ER. Role of neutrophil apoptosis in the resolution of pulmonary inflammation. Monaldi Arch Chest Dis 1999; 54: $345-349$.
13. Wouters EF, Creutzberg EC, Schols AM. Systemic effects in COPD. Chest 2002; 121: Suppl. 5, 127S-130S.

14. Biffl WL, Moore EE, Moore FA, Barnett CC Jr. Interleukin6 suppression of neutrophil apoptosis is neutrophil concentration dependent. J Leukoc Biol 1995; 58: 582-584.

15. Wedzicha JA, Seemungal TA, MacCallum PK, et al. Acute exacerbations of chronic obstructive pulmonary disease are accompanied by elevations of plasma fibrinogen and serum IL-6 levels. Thromb Haemost 2000; 84: 210-215.

16. Turlej RK, Fievez L, Sandersen CF, et al. Enhanced survival of lung granulocytes in an animal model of asthma: evidence for a role of GM-CSF activated STAT5 signalling pathway. Thorax 2001; 56: 696-702.

17. Grutkoski PS, Graeber CT, Ayala A, Simms HH. Paracrine suppression of apoptosis by cytokine-stimulated neutrophils involves divergent regulation of NF-kappaB, Bcl-X(L), and Bak. Shock 2002; 17: 47-54.

18. Aoshiba K, Nagai A, Yasui S, Konno K. Nicotine prolongs neutrophil survival by suppressing apoptosis. J Lab Clin Med 1996; 127: 186-94.

19. Ruiz LM, Bedoya G, Salazar J, Garcia de OD, Patino PJ. Dexamethasone inhibits apoptosis of human neutrophils induced by reactive oxygen species. Inflammation 2002; 26: 215-222.

20. Sunil VR, Connor AJ, Zhou P, Gordon MK, Laskin JD, Laskin DL. Activation of adherent vascular neutrophils in the lung during acute endotoxemia. Respir Res 2002; 3: 21.

21. Saba S, Soong G, Greenberg S, Prince A. Bacterial stimulation of epithelial G-CSF and GM-CSF expression promotes PMN survival in CF airways. Am J Respir Cell Mol Biol 2002; 27: 561-567. 